\title{
Association of serum angiopoietin-like protein 2 with carotid intima-media thickness in subjects with type 2 diabetes
}

Chang Hee Jung ${ }^{1 \dagger}$, Woo Je Lee ${ }^{1 \dagger}$, Min Jung Lee ${ }^{1}$, Yu Mi Kang ${ }^{1}$, Jung Eun Jang ${ }^{1}$, Jaechan Leem ${ }^{1}$, Yoo La Lee ${ }^{2}$, So Mi Seol ${ }^{2}$, Hae Kyeong Yoon ${ }^{2}$ and Joong-Yeol Park ${ }^{1,3^{*}}$

\begin{abstract}
Background: Although recent animal studies have suggested that angiopoietin-like protein 2 (ANGPTL2), a novel inflammatory adipokine, is likely to be involved in the pathogenesis of atherosclerosis, in rodents, little is known regarding whether serum ANGPTL2 level is also associated with atherosclerosis in humans, especially in patients with type 2 diabetes. The aim of this study was to investigate whether serum ANGPTL2 concentration is associated with atherosclerosis by measuring carotid intima-media thickness (IMT) in subjects with type 2 diabetes without previous history of cardiovascular diseases. In addition, we examined the clinical and biochemical variables associated with serum ANGPLT2 concentration.

Methods: We measured the circulating ANGPTL2 level in 166 subjects (92 men and 74 women; mean age of 60.0 years) with type 2 diabetes. Measurements of carotid IMT were performed in all subjects.

Results: Serum ANGPTL2 concentration was positively correlated with carotid IMT ( $r=0.220, p=0.004)$. In multiple linear regression, serum ANGPTL2 concentration was independently associated with increased carotid IMT along with older age, male gender, and higher systolic blood pressure. Higher levels of hemoglobin A1c and high-sensitivity C-reactive protein were significantly associated with elevated serum ANGPTL2 concentration in subjects with type 2 diabetes.
\end{abstract}

Conclusions: Serum ANGPTL2 concentration was significantly and positively associated with carotid atherosclerosis in patients with type 2 diabetes, suggesting that ANGPTL2 may be important in the atherosclerosis in humans.

Keywords: Angiopoietin-like protein 2, Atherosclerosis, Carotid, Intima media thickness, Type 2 diabetes

\section{Background}

Obesity is now clearly associated with a state of chronic low-grade inflammation characterized by abnormal cytokine production and activation of inflammatory signaling pathways in adipose tissues, which contributes to insulin resistance [1]. Angiopoietin is a part of a family of vascular growth factors that are important modulators of angiogenesis [2]. A family of proteins structurally similar to angiopoietin was identified and designated as 'angiopoietin-like proteins (ANGPTLs)' [3-7]. Encoded by

\footnotetext{
* Correspondence: jypark@amc.seoul.kr

${ }^{\dagger}$ Equal contributors

${ }^{1}$ Asan Diabetes Center, University of Ulsan College of Medicine, Seoul, Republic of Korea

${ }^{3}$ Department of Internal Medicine, Asan Medical Center, University of Ulsan College of Medicine, Poongnap-dong, Songpa-gu, Seoul 138-736, Republic of Korea

Full list of author information is available at the end of the article
}

eight genes, ANGPTL1-8 are structurally similar to angiopoietins, characterized by a coiled-coil domain in the $\mathrm{N}$-terminus and a fibrinogen-like domain in the $\mathrm{C}$ terminus, except for ANGPTL8 which does not have a fibrinogen-like domain [3-7]. However, ANGPTLs do not bind to angiopoietin receptors, (i.e., either Tie2 or the related protein Tie1), suggesting that these ligands function differently from angiopoietins [6,8,9]. Several studies have reported that a subset of ANGPTLs function in glucose, lipid, and energy metabolism, although they mainly regulate angiogenesis $[3,7]$.

Among ANGPTLs, ANGPTL2 is abundantly expressed in visceral adipose tissue and has been demonstrated to be involved in adipose tissue remodeling, such as angiogenesis and extracellular matrix remodeling, ultimately leading to adipogenesis and adipocyte hypertrophy, increasing excess energy storage in adipose tissue $[4,10]$. In cases of

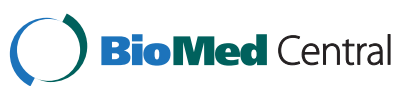


severe obesity, ANGPTL2 expression is abundant in adipose tissues and excess signaling by ANGPTLs leads to chronic inflammation, resulting in metabolic diseases such as obesity-related insulin resistance and type 2 diabetes $[4,10]$. Besides its metabolic role, ANGPTL2 has growingly received attention due to its deleterious effect on atherosclerotic diseases [9-14]. In a mouse model, ANGPTL2-deficient animals showed decreased abdominal aortic aneurysms, characterized pathologically by atherosclerotic changes accompanying chronic inflammation and infiltrating inflammatory cells [13]. In addition, perivascular ANGPTL2 accelerates neointimal hyperplasia after endovascular injury in mice [12]. Recently, disruption of ANGPTL2 in apolipoprotein E-deficient mice $\left(\mathrm{ApoE}^{-1-}\right.$ / Angptl $^{-/}$) was found to attenuate atherosclerosis progression by decreasing the number of macrophage infiltrating atheromatous plaques, thereby reducing vascular inflammation [9]. Although these findings support the concept that ANGPTL2 is more likely to be involved in the regulation of vascular function, more specifically in atherosclerosis in rodents $[9,10,12-14]$, few studies have examined the relevance of circulating ANGPTL2 levels to subclinical atherosclerosis in humans [9]. Furthermore, it remains unclear about to what extent ANGPTL2 contributes to the development of atherosclerosis independently of conventional cardiovascular risk factors, especially in subjects with type 2 diabetes.

In our current study, we investigated whether serum ANGPTL2 concentration was associated with the parameters of atherosclerosis in subjects with type 2 diabetes using carotid artery intima-media thickness (IMT), a noninvasive index of early atherosclerosis [15]. In addition, we tried to identify the clinical and biochemical variables related to the serum concentration of ANGPTL2.

\section{Methods}

Study populations

As previously described [16], we consecutively recruited patients who visited the Asan Diabetes Center at Asan Medical Center (AMC), Seoul, Republic of Korea between November 2012 and January 2014 for treatment of diabetes and evaluation of micro- and macrovascular complications of diabetes. Before starting the recruitment process, the study was registered at the Clinical Research Information Service (cris.nih.go.kr) (KCT0000598). Our study population comprised 203 subjects who had undergone carotid artery ultrasonography during recruitment.

A single specially trained nurse interviewed all participants and obtained information on medications and history of previous medical or surgical diseases. A history of cardiovascular disease (CVD) was defined as a history of physician-diagnosed CVD (eg, previous myocardial infarction, angina, coronary-artery bypass grafting, and/or stroke). After exclusion of subjects with a history of CVD $(\mathrm{n}=37)$, a total of 166 subjects (92 men aged 38-78 years [mean age, 59.7 years] and 74 women aged $38-76$ years [mean age, 60.4 years]) with type 2 diabetes were included in the study. All subjects provided written informed consent and the study was approved by the Institutional Review Board of the AMC.

Antidiabetic treatments were categorized as none, oral hypoglycemic agents (OHAs), or insulin with or without OHAs. Peroxisome proliferator-activated receptor $\gamma$ agonists such as thiazolidinedione affect the expression of ANGPTL2 [10], therefore patients treated with thiazolidinedione were excluded from the study prior to the initiation of the recruitment process. Antihypertensive medications included any of the following: angiotensin-converting enzyme inhibitors, angiotensin II receptor blockers, $\beta$ blockers, calcium-channel blockers, diuretics, or $\alpha$-blockers.

\section{Anthropometric and laboratory measurements}

Height and weight were obtained while subjects wore light clothing without shoes. The waist circumference (WC, cm) was measured midway between the costal margin and the iliac crest at the end of a normal expiration. The blood pressure (BP) was measured on the right arm after a rest of $\geq 5 \mathrm{~min}$.

After overnight fasting, early morning blood samples were drawn from the antecubital vein into vacuum tubes and subsequently analyzed by a central, certified laboratory at the AMC. Measurements included fasting plasma glucose (FPG), hemoglobin A1c (HbA1c), insulin, highsensitivity C-reactive protein (hsCRP), several lipid parameters, and liver enzyme levels.

FPG was measured by the glucose oxidase method using a Toshiba 200FR Neo (Toshiba Medical Systems Co., Ltd., Tokyo, Japan). HbA1c was measured by high-performance liquid chromatography (HPLC) using a Variant II Turbo (Bio-Rad Laboratories, Hercules, CA). Fasting total cholesterol, high-density lipoprotein-cholesterol (HDL-C), lowdensity lipoprotein-cholesterol (LDL-C), triglyceride (TG), uric acid, aspartate aminotransferase (AST), and alanine aminotransferase (ALT) were measured using an enzymatic colorimetric method (Toshiba Medical Systems). HsCRP was measured using the immunoturbidimetric method (Toshiba Medical Systems). Serum insulin was measured by immunoradiometric assay (TFB Co., Ltd, Tokyo, Japan). Homeostatic model assessment of insulin resistance (HOMA-IR) was used as a surrogate measure of systemic insulin resistance, which has been suggested to be a useful test for the evaluation of insulin resistance even in patients with type 2 diabetes treated with an insulin secretagogue or insulin $[17,18]$. All enzyme activities were measured at $37^{\circ} \mathrm{C}$. HOMA-IR was calculated according to the following equation: HOMA-IR $=[$ Insulin $(\mu \mathrm{U} / \mathrm{mL}) \times$ FPG (mmol/L)]/22.5. 
The extent of albuminuria was determined from urinary albumin-to-creatinine ratio (UACR), which was measured by a photometric method using the Integra 800 system (Roche Diagnostics, Indianapolis, IN) in a random spot urine collection. Creatinine was measured using the Jaffe method, and estimated glomerular filtration rate (eGFR) was calculated using the modified Modification of Diet in Renal Disease (MDRD) equation [19].

\section{Measurement of serum concentrations of ANGPTL 2 and total adiponectin}

Fasting venous blood samples were centrifuged, and the supernatants were carefully collected to exclude cell components. All samples with hemolysis or clotting were discarded. Serum samples were stored at $-80^{\circ} \mathrm{C}$ until use. Serum ANGPTL2 concentrations were measured using a human ANGPTL2 sandwich enzyme-linked immunosorbent assay (ELISA) kit (Immuno-Biological Laboratories Co., Ltd, Japan) with two mouse monoclonal antibodies that were confirmed to recognize only ANGPTL2 and not react with any other ANGPTLs or angiopoietins [10]. The kit's lower detection limit is typically less than $0.01 \mathrm{ng} / \mathrm{ml}$. The intra-and inter-assay coefficients of variations (CVs) of the ELISA kit were $<5.9 \%$ and $<10.5 \%$, respectively. Serum total adiponectin concentration was also measured using commercially available ELISA kits (Adipogen, Seoul, South Korea), with intra-and inter-assay CVs of $<4.0 \%$ and $<6.0 \%$, respectively. Samples were assayed in duplicate and all results were reported as means.

\section{Ultrasonographic measurement of carotid IMT}

Ultrasound analysis was performed by a single specialized technician who was blind to the patient's clinical data. The examination was carried out according to a previously established protocol standardized for both carotid arteries [20]. Images were acquired at end diastole (defined as the $\mathrm{R}$ wave of the electrocardiogram) using a 5-12 $\mathrm{MHz}$ linear transducer and high resolution ultrasound (HD 11 XE, Philips Healthcare, Andover MA). All measurements were recorded with the subject in a supine position with the head elevated to $45^{\circ}$ and tilted to either side by $30^{\circ}$. The IMT was measured over a $1-\mathrm{cm}$ segment of the artery located approximately $0.5 \mathrm{~cm}$ below the carotid-artery bulb and considered not to contain any plaque (i.e., not to have any perceivable protrusion of the artery wall into the lumen) [21,22]. For the purpose of the present study, a QLAB IMT-quantification software measurement plug-in (Philips Healthcare) was used to increase the consistency and reliability of IMT measurements, reduce the effort required to successfully carry out IMT measurements, and minimize the time needed to complete an IMT study [23]. The correlation coefficient for carotid IMT was 0.985 . To quantify carotid artery wall thickness we used the mean of the right and left IMTs in the present study.

\section{Statistical analysis}

Continuous variables with normal distribution were expressed as mean $\pm \mathrm{SD}$, whereas continuous variables with skewed distribution were expressed as median (and interquartile range). Categorical variables were expressed as proportions (\%). The serum ANGPTL2 tertiles were as follows: $\mathrm{Q} 1 \leq 3.27 \mathrm{ng} / \mathrm{mL}, \mathrm{Q} 2=3.28-5.19 \mathrm{ng} / \mathrm{mL}$, and Q3 $\geq 5.20 \mathrm{ng} / \mathrm{mL}$. Demographic and biochemical characteristics of the study population according to the serum ANGPTL2 tertiles were compared using a one-way analysis of variance (ANOVA) with the Scheffe's method as a post-hoc analysis, or the Kruskal-Wallis test with the Dunn procedure for continuous variables, and the chisquared test for categorical variables. The correlation between serum ANGPTL2 and various parameters, including carotid IMT, was evaluated by Pearson correlation analysis. Logarithmic (log) transformation of values showing a skewed distribution was carried out before the correlation analysis. The association between the mean carotid IMT and serum ANGPTL2 level was investigated using backward multiple linear regression, with mean carotid IMT serving as the dependent variable. To identify the independent determinants of carotid IMT, univariate regression analysis was performed as a preliminary step to a multiple linear regression. In the multivariate model, all the variables that were associated with the carotid IMT with $p$ values $<0.20$ in the univariate regression analysis were included. To identify the factors contributing to increased serum ANGPTL2 levels, a backward multiple linear regression was performed with serum ANGPTL2 levels serving as the dependent variable. This analysis was adjusted for variables, which were associated with serum ANGPTL2 with $p$ values $<0.20$ in the univariate regression analysis. All independent variables in the multiple linear regression analysis were tested for multicollinearity; if the variance inflation factor (VIF) exceeded 10, the variable was considered to be collinear. All statistical analyses were carried out with SPSS version 19.0 for Windows (IBM Corp., Armonk, NY). A two-tailed $p$-value $<0.05$ was regarded as statistically significant

\section{Results}

The average age of the study subjects was $60.0 \pm 8.3$ years, and about $60 \%$ of subjects were obese with a mean BMI of $26.0 \pm 3.4 \mathrm{~kg} / \mathrm{m}^{2}$ [24]. The mean concentration of serum ANGPTL2 was $4.75 \pm 2.63 \mathrm{ng} / \mathrm{ml}$. The clinical and biochemical characteristics of the study subjects according to the serum ANGPTL2 tertile are shown in Table 1. A number of variables, including age, sex, BMI, WC, systolic BP, diastolic BP, current smoker, duration 
Table 1 Clinical biochemical characteristics of the study subjects according to the serum ANGPTL2 tertile

\begin{tabular}{|c|c|c|c|c|}
\hline \multirow[b]{3}{*}{ Variables } & \multicolumn{3}{|c|}{ Serum ANGPTL2 tertile } & \multirow{3}{*}{$P$ value } \\
\hline & Q1 & Q2 & Q3 & \\
\hline & $(\leq 3.27 \mathrm{ng} / \mathrm{ml})$ & $(3.28 \sim 5.19 \mathrm{ng} / \mathrm{ml})$ & $(\leq 5.20 \mathrm{ng} / \mathrm{ml})$ & \\
\hline $\mathrm{N}$ & 55 & 56 & 55 & - \\
\hline Age (years) & $60.3 \pm 7.1$ & $60.8 \pm 8.3$ & $58.9 \pm 9.4$ & 0.429 \\
\hline Sex (male, \%) & 65.5 & 44.6 & 56.4 & 0.085 \\
\hline BMI $\left(\mathrm{kg} / \mathrm{m}^{2}\right)$ & $25.3 \pm 2.7$ & $26.5 \pm 3.6$ & $26.2 \pm 3.7$ & 0.155 \\
\hline WC $(\mathrm{cm})$ & $88.4 \pm 9.6$ & $91.0 \pm 9.0$ & $90.2 \pm 7.7$ & 0.281 \\
\hline Systolic BP (mmHg) & $126.9 \pm 17.0$ & $126.8 \pm 15.5$ & $128.4 \pm 14.1$ & 0.823 \\
\hline Diastolic BP (mmHg) & $75.2 \pm 9.1$ & $74.6 \pm 8.4$ & $76.2 \pm 8.5$ & 0.625 \\
\hline Current smoker (\%) & 21.8 & 12.5 & 20.0 & 0.383 \\
\hline Duration of diabetes (years) & $12(4-14)$ & $9(6-14)$ & $8(4-13)$ & 0.225 \\
\hline Antidiabetic medication (\%) & & & & 0.400 \\
\hline None & 7.3 & 1.8 & 7.3 & \\
\hline $\mathrm{OHA}$ & 74.5 & 85.7 & 81.8 & \\
\hline Insulin $\pm \mathrm{OHA}$ & 18.2 & 12.5 & 10.9 & \\
\hline Antihypertensive medication (\%) & 52.7 & 66.1 & 56.4 & 0.336 \\
\hline Statin use (\%) & 76.4 & 71.4 & 76.4 & 0.790 \\
\hline FPG (mmol/L) & $7.4 \pm 2.0^{b}$ & $7.8 \pm 1.8^{b, c}$ & $8.2 \pm 2.0^{c}$ & 0.042 \\
\hline $\mathrm{HbA1c}(\%)$ & $6.8(6.4-7.2)^{b}$ & $7.0(6.3-7.9)^{b, c}$ & $7.3(6.7-8.2)^{c}$ & 0.047 \\
\hline $\mathrm{HbA1c}(\mathrm{mmol} / \mathrm{mol})$ & $51(46-55)^{b}$ & $53(45-63)^{\mathrm{b}, \mathrm{c}}$ & $56(50-66)^{c}$ & 0.047 \\
\hline Total cholesterol (mmol/L) & $4.1 \pm 1.0$ & $4.0 \pm 0.8$ & $4.0 \pm 0.8$ & 0.615 \\
\hline TG $(\mathrm{mmol} / \mathrm{L})$ & $1.1(0.9-2.1)$ & $1.4(1.1-2.2)$ & $1.6(1.1-2.4)$ & 0.037 \\
\hline LDL-C (mmol/L) & $2.5 \pm 0.7$ & $2.5 \pm 0.7$ & $2.5 \pm 0.7$ & 0.897 \\
\hline $\mathrm{HDL}-\mathrm{C}(\mathrm{mmol} / \mathrm{L})$ & $1.3 \pm 0.3^{b}$ & $1.2 \pm 0.3^{\mathrm{b}, \mathrm{c}}$ & $1.1 \pm 0.3^{c}$ & 0.036 \\
\hline Uric acid ( $\mu \mathrm{mol} / \mathrm{L})$ & $297.4 \pm 89.2$ & $285.5 \pm 71.4$ & $297.4 \pm 71.4$ & 0.781 \\
\hline AST (U/L) & $21(18-26)$ & $23(20-31)$ & $23(20-34)$ & 0.079 \\
\hline $\operatorname{ALT}(U / L)$ & $18(14-25)$ & $21(16-38)$ & $20(15-33)$ & 0.205 \\
\hline hsCRP (mg/L) & $0.5(0.1-1.1)$ & $0.6(0.3-1.1)$ & $0.6(0.4-1.6)$ & 0.130 \\
\hline UACR (mg/g) & $7.0(4.0-24.8)$ & $12.8(5.7-23.9)$ & $9.3(4.9-26.7)$ & 0.440 \\
\hline eGFR $\left(\mathrm{ml} / \mathrm{min} / 1.73 \mathrm{~m}^{2}\right)$ & $91.8 \pm 22.5$ & $94.6 \pm 22.1$ & $91.8 \pm 20.6$ & 0.726 \\
\hline HOMA-IR & $2.2(1.2-3.7)^{b}$ & $2.5(1.6-4.5)^{b, c}$ & $2.9(1.7-5.3)^{c}$ & 0.027 \\
\hline Adiponectin $(\mu \mathrm{g} / \mathrm{ml})$ & $9.1 \pm 5.4$ & $9.9 \pm 7.3$ & $8.5 \pm 4.7$ & 0.465 \\
\hline Mean IMT (mm) & $0.66 \pm 0.12^{b}$ & $0.67 \pm 0.10^{b}$ & $0.73 \pm 0.12$ & 0.001 \\
\hline
\end{tabular}

adifference among three groups.

$\mathrm{b}, \mathrm{c}$ the same letters indicate a statistically insignificant difference.

of diabetes, antidiabetic medications, percentage of subjects taking antihypertensive medications or statins, levels of total cholesterol, LDL-C, uric acid, AST, ALT, hsCRP, UACR, eGFR, and total adiponectin, were similar among the three groups (Table 1). FPG, HbA1c, TG, HOMA-IR, and carotid IMT linearly increased ( $p$ value 0.047 to 0.001 ), while HDL-C decreased linearly across increasing serum ANGPTL2 tertile categories $(p=0.036)$.

Table 2 shows the correlation between serum levels of ANGPTL2 versus various glucometabolic parameters. In the Pearson's correlation analysis, the ANGPTL2 levels showed a positive correlation with AST $(r=0.222, p=0.004)$, hsCRP $(r=0.218, p=0.011)$, HbA1c $(r=0.178, p=0.022)$, and HOMA-IR $(r=0.116, p=0.045)$ following $\log$ transformation of all four factors.

In the Pearson's correlation analysis, the ANGPTL2 level was positively correlated with carotid IMT $(r=0.220$, $p=0.004$, Figure 1). The correlation between serum ANGPTL2 level and carotid IMT was significant even after adjusting for age $(r=0.272, p<0.001)$.

As shown in Table 3, multiple linear regression demonstrated that serum ANGPTL2 $[B(\mathrm{SE})=0.012(0.003)$; 
Table 2 Correlation between serum levels of ANGPTL2 and glucometabolic parameters

\begin{tabular}{|c|c|c|}
\hline \multirow[b]{2}{*}{ Variables } & \multicolumn{2}{|c|}{ ANGPTL2 (ng/ml) } \\
\hline & $r$ & $P$ value \\
\hline BMI & 0.093 & 0.231 \\
\hline WC & 0.047 & 0.551 \\
\hline SBP & 0.003 & 0.968 \\
\hline DBP & 0.014 & 0.859 \\
\hline FPG & 0.144 & 0.064 \\
\hline $\mathrm{HbA} 1 \mathrm{c}^{\mathrm{a}}$ & 0.178 & 0.022 \\
\hline Duration of diabetes & -0.136 & 0.080 \\
\hline Total cholesterol & -0.021 & 0.786 \\
\hline $\mathrm{TG}^{\mathrm{a}}$ & 0.148 & 0.057 \\
\hline LDL-C & -0.005 & 0.949 \\
\hline $\mathrm{HDL}-\mathrm{C}$ & -0.137 & 0.078 \\
\hline Uric acid & -0.026 & 0.740 \\
\hline $\mathrm{AST}^{\mathrm{a}}$ & 0.222 & 0.004 \\
\hline $\mathrm{ALT}^{\mathrm{a}}$ & 0.142 & 0.068 \\
\hline hsCRPa & 0.218 & 0.011 \\
\hline UACR & 0.084 & 0.281 \\
\hline eGFR & -0.056 & 0.475 \\
\hline HOMA-IR ${ }^{a}$ & 0.116 & 0.045 \\
\hline Adiponectin & -0.096 & 0.235 \\
\hline
\end{tabular}

aLogarithmic transformation was performed. NS: not significant.

$p<0.001]$ and other clinical parameters, including age $[B$ $(\mathrm{SE})=0.005$ (0.001); $p<0.001$ ], male gender [(vs. female), $B$ $(\mathrm{SE})=0.060(0.015) ; p<0.001]$, and systolic BP $[B(\mathrm{SE})=$ 0.001 (0.001); $p=0.016$ ], were independently associated with carotid IMT.

Finally, we examined the clinical and biochemical factors that contributed to the elevated serum ANGPTL2 concentrations in our subjects with type 2 diabetes. Multiple linear regression demonstrated that higher HbA1c

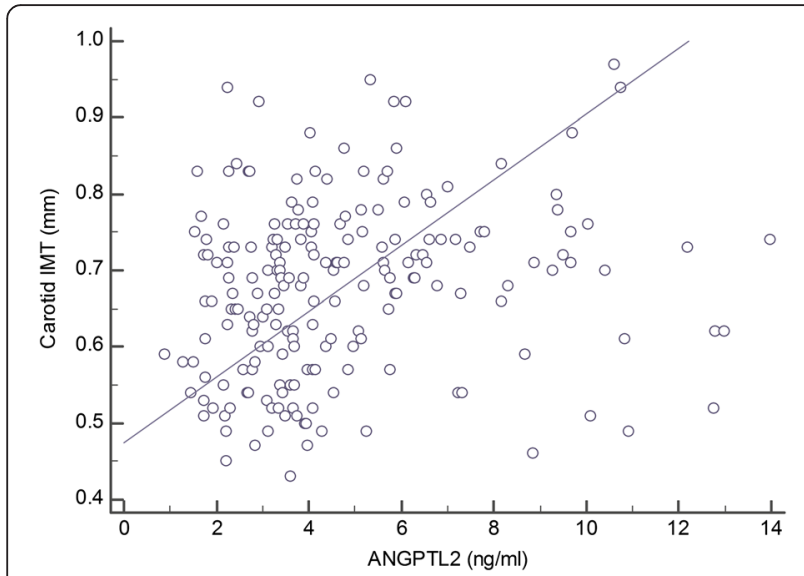

Figure 1 Correlation between the serum ANGPLT2 concentrations and carotid IMT $(r=0.220, p=0.004)$.
$[B(\mathrm{SE})=3.648(1.789) ; p=0.043]$ and hsCRP $[B(\mathrm{SE})=$ 0.439 (0.196); $p=0.027]$ were significantly associated with elevated serum ANGPTL2 concentrations in these individuals (Additional file 1: Table S1).

\section{Discussion}

ANGPTL2 is a novel proinflammatory cytokine primarily secreted by adipose tissues, although vascular endothelial cells and monocyte/macrophages also secrete this protein $[9,10,13]$. ANGPTL2 has been known to regulate angiogenesis similarly to several other ANGPTLs [3,4]. In addition, ANGPTL2 has the unique capacity to induce an inflammatory response in blood vessels as well as in adipose tissue $[9,10,12-14]$. For instance, several experimental studies have suggested the biological relevance of ANGPTL2 as a regulator of atherosclerosis in rodents [9,10,12-14]. In our current study, the serum ANGPTL2 concentration is significantly and positively associated with carotid IMT in subjects with type 2 diabetes $(r=0.220, p<0.01$, Figure 1$)$. Furthermore, this association is independent of conventional cardiovascular risk factors (Table 3). Considering the reported harmful effects of ANGPTL2 on vascular inflammation in rodents $[9,10,12-14]$, our current findings provide further evidence that this protein may play a key role in atherosclerosis in humans.

To date, there have been a few studies that have measured the serum ANGPTL2 concentrations in human subjects $[9,10,14,25-27]$ and investigated the differences in these levels according to the presence of CVD $[9,10,14]$. In 2009 , Tabata et al. measured the serum levels of ANGPTL2 in a total of 109 patients with coronary heart disease (CHD) diagnosed by coronary angiography and 89 drug-naïve type 2 diabetes patients [10]. These authors demonstrated that serum ANGPTL2 was significantly increased in patients with CHD or type 2 diabetes [10]. More recently, Horio et al. demonstrated that ANGPTL2 was abundantly expressed in atheromatous plaques in human coronary artery, and that the circulating ANGPTL2 levels measured by ELISA were higher in human subjects with CHD (defined by coronary artery stenosis $\geq 75 \%$ on coronary angiography; $\mathrm{n}=89$ ) compared with age-matched non-CHD subjects $(n=30)$ [9]. Furthermore, they showed that serum ANGPTL2 levels were associated with carotid IMT in 359 seniors aged between 85 to 99 years with no history of CVD, but with relatively reduced eGFR (mean $62.5 \pm 16.8 \mathrm{ml} / \mathrm{min} / 1.73 \mathrm{~m}^{2}$ ) and advanced stages of atherosclerosis (mean carotid IMT of $0.99 \pm 0.15 \mathrm{~mm}$ compared with our current study measurement of $0.69 \pm 0.12 \mathrm{~mm}$ ), among whom a small proportion of subjects with diabetes was included (approximately 18.4\%) [9]. Although these previous data support the hypothesis that serum ANGPTL2 is strongly related to atherosclerosis in humans, little is known whether this role of 
Table 3 Multiple linear regression with the mean carotid IMT as a dependent variable

\begin{tabular}{|c|c|c|c|c|c|c|}
\hline \multirow[b]{2}{*}{ Variables } & \multirow[b]{2}{*}{$\beta$} & \multicolumn{2}{|c|}{ Univariate analysis } & \multirow[b]{2}{*}{$\beta$} & \multicolumn{2}{|c|}{ Multivariate analysis } \\
\hline & & $B(\mathrm{SE})$ & $P$ value & & $B(\mathrm{SE})$ & $P$ value \\
\hline Age (years) & 0.376 & $0.005(0.001)$ & $<0.001$ & 0.374 & $0.005(0.001)$ & $<0.001$ \\
\hline Male (vs. female) & 0.235 & $0.054(0.018)$ & 0.002 & 0.257 & $0.060(0.015)$ & $<0.001$ \\
\hline BMI & 0.093 & $0.003(0.003)$ & 0.234 & & & \\
\hline WC & 0.176 & $0.002(0.001)$ & 0.023 & & & \\
\hline Systolic BP & 0.242 & $0.002(0.001)$ & 0.002 & 0.165 & $0.001(0.001)$ & 0.016 \\
\hline Diastolic BP & 0.177 & $0.002(0.001)$ & 0.023 & & & \\
\hline Current smoker $^{a}$ & -0.009 & $-0.003(0.023)$ & 0.908 & & & \\
\hline Duration of diabetes (years) & 0.146 & $0.003(0.001)$ & 0.060 & & & \\
\hline HTN medication use ${ }^{b}$ & 0.185 & $0.043(0.018)$ & 0.017 & & & \\
\hline Statin use $\mathrm{b}^{\mathrm{b}}$ & -0.060 & $-0.016(0.021)$ & 0.443 & & & \\
\hline FPG & 0.076 & $0.000(0.000)$ & 0.329 & & & \\
\hline $\mathrm{HbA} 1 \mathrm{c}^{\mathrm{c}}$ & 0.089 & $0.080(0.070)$ & 0.253 & & & \\
\hline Total cholesterol & -0.006 & $0.000(0.000)$ & 0.938 & & & \\
\hline $\mathrm{TG}^{\mathrm{d}}$ & -0.026 & $-0.006(0.018)$ & 0.737 & & & \\
\hline LDL-C & 0.000 & $0.000(0.000)$ & 0.995 & & & \\
\hline $\mathrm{HDL}-\mathrm{C}$ & -0.048 & $0.000(0.001)$ & 0.538 & & & \\
\hline Uric acid & 0.162 & $0.014(0.007)$ & 0.037 & & & \\
\hline $\mathrm{AST}^{\mathrm{C}}$ & 0.044 & $0.014(0.024)$ & 0.572 & & & \\
\hline$A L T^{c}$ & 0.051 & $0.011(0.016)$ & 0.511 & & & \\
\hline hsCRPc & 0.057 & $0.006(0.009)$ & 0.513 & & & \\
\hline UACR & -0.004 & $0.000(0.007)$ & 0.958 & & & \\
\hline eGFR & -0.153 & $-0.001(0.000)$ & 0.049 & & & \\
\hline HOMA-IR & -0.029 & $-0.003(0.010)$ & 0.730 & & & \\
\hline Adiponectin & -0.095 & $-0.002(0.002)$ & 0.224 & & & \\
\hline Serum ANGPTL2 & 0.220 & $0.010(0.003)$ & 0.004 & 0.263 & $0.012(0.003)$ & $<0.001$ \\
\hline
\end{tabular}

$\beta$ : standardized coefficient, B: unstandardized coefficient, SE: standard error.

${ }^{\mathrm{a}}$ vs. noncurrent smoker.

bs. nonuser.

'Logarithmic transformation was performed.

ANGPTL2 applies in purely type 2 diabetes cases and/or in subject with early stage atherosclerosis. Our current evidence may suggest the independent involvement of ANGPTL2 in the pathogenesis of early subclinical atherosclerosis in humans, especially in subjects with type 2 diabetes. This role of ANGPTL2 in early atherosclerosis accords with the findings of a previous animal study, in which ANGPTL2 altered the release of nitric oxide, an important anti-atherogenic molecule [28], leading to endothelial dysfunction at stages of atherosclerosis preceding atherosclerotic plaque formation $[9,14]$

In humans, the ANGPTL2 concentration in the circulation is up-regulated in obesity (particularly visceral obesity) and is correlated with the levels of systemic insulin resistance $[10,25]$. In accordance with these previous results $[10,25]$, biochemical variables indicating insulinresistant profiles such as higher TG and HOMA-IR and lower HDL-C could be seen across increasing serum ANGPTL2 tertile categories in our present analyses (Table 1). In addition, we found a significant positive correlation between serum ANGPTL2 concentration and AST, hsCRP, and HOMA-IR (Table 2). Our current findings thus support the previously suggested possibility that circulating ANGPTL2 levels can serve as a marker of obesity-induced metabolic abnormalities as well as atherosclerosis [10,25,29].

There have been conflicting results regarding the role of ANGPTL2 in glucose metabolism [10,30]. In a previous study using ANGPTL2 knock-out mice, insulin sensitivity was improved in both the skeletal muscle and liver in mice fed with a high-fat diet [10]. In contrast, the replenishment of ANGPTL2 stimulated insulin sensitivity and improved glucose tolerance in mice [30]. In our current study, glycemic variables including FPG and 
HbA1c increased as serum ANGPTL2 tertile categories increased (Table 1), and there was a positive correlation observed between the serum ANGPTL2 concentration and HbA1c (Table 2), which are in line with the results of a previous study [25]. Furthermore, we observed that $\mathrm{HbA1c}$ is one of the independent contributors to the increased serum ANGPTL2 concentration (Additional file 1: Table S1). Recently, Doi et al. firstly demonstrated that elevated serum ANGPTL2 levels were positively associated with the development of type 2 diabetes in a total of 2,164 community-dwelling Japanese individuals, independent of other risk factors including hsCRP levels [31]. In that analysis, the risk of developing type 2 diabetes was significantly higher in the highest ANGPTL2 quartile (i.e., $\geq 3.41 \mathrm{ng} / \mathrm{ml}$ ) than in the lowest quartile (hazard ratio, 1.80) [31]. Although further studies are needed to reveal the role of ANGPTL2 in the pathogenesis of type 2 diabetes, these earlier results and our present findings suggest the possibility that ANGPTL2 might be one of key mediators that link obesity and type 2 diabetes.

It is well accepted that obesity can be regarded as a state of chronic subclinical inflammation [32-34], and levels of hsCRP, a marker of low-grade inflammation, have been previously linked to visceral obesity $[35,36]$. The liver is assumed to be the major source of CRP production; however, adipose tissues have also been suggested as a direct source of CRP $[37,38]$. In our current study, the serum ANGPTL2 concentration showed a significant correlation with hsCRP (Table 2), and hsCRP was one of the independent contributors to the increased serum ANGPTL2 concentration (Additional file 1: Table S1), which are in accordance with the results of a previous study [10]. This close relationship between ANGPTL2 and hsCRP further implicates that ANGPTL2 is one of key adipocyte-derived inflammatory mediators linking obesity to related metabolic diseases.

This study has several noteworthy limitations. First, our sample size was not large enough to make any definite conclusions. This limitation was partly reflected in the results of our study, in which there was an insignificant inverse correlation between the levels of total serum adiponectin and ANGPTL2 ( $r=-0.096, p=0.235$ in Table 2), which is in contrast to the results of a previous study showing a significant association between these two factors [39]. Second, we lacked data on other inflammatory markers of systemic inflammation besides hsCRP. Additional markers reflecting adipose tissue dysfunction such as IL-6 would significantly strengthen the findings of this study [40]. Third, due to the cross-sectional design of our present study, we could not assess the temporal relationship between the serum ANGPTL2 level and increased carotid atherosclerosis. Fourth, because we included only Korean subjects, our results may not be applicable to other ethnic populations.
Despite these limitations, our current study is meaningful in that we demonstrated the relationships between serum ANGPTL2 concentration and early subclinical atherosclerosis in humans, especially in subjects with type 2 diabetes.

\section{Conclusions}

In conclusion, we found that the serum ANGPTL2 concentration is significantly and positively associated with carotid IMT in human subjects with type 2 diabetes. This further supports the recently proposed notion that ANGPTL2 may be an important factor in the pathogenesis of atherosclerosis in humans $[9,10,14]$, similarly to its possible role in the regulation of vascular function in rodents [9,10,12-14]. Further prospective studies are needed to determine whether ANGPTL2 is a useful predictive marker for CVD.

\section{Additional file}

Additional file 1: Table S1. Univariate and multivariate analysis of the relationship between the serum ANGPTL2 concentrations and various clinical parameters.

\section{Abbreviations}

ALT: Alanine aminotransferase; AMC: Asan medical center

ANGPTL: Angiopoietin-like protein; ANOVA: One-way analysis of variance; AST: Aspartate aminotransferase; BP: Blood pressure; CHD: Coronary heart disease; CV: Coefficients of variation; CVD: Cardiovascular disease; eGFR: Estimated glomerular filtration rate; ELISA: Enzyme-linked immunosorbent assay; FPG: Fasting plasma glucose; HbA1c: Hemoglobin A1c; HDL-C: High-density lipoprotein-cholesterol; HOMA-IR: Homeostatic model assessment of insulin resistance; HPLC: High-performance liquid chromatography; hsCRP: High-sensitivity C-reactive protein; IMT: Intima-media thickness; LDL-C: Low-density lipoprotein-cholesterol; MDRD: Modified modification of diet in renal disease; OHAs: Oral hypoglycemic agents; TG: Triglyceride; UACR: Urinary albumin-to-creatinine ratio; VIF: Variance inflation factor; WC: Waist circumference.

\section{Competing interests}

The authors declare that they have no competing interests.

\section{Authors' contributions}

$\mathrm{CHJ}$ and WJL researched data and wrote the manuscript. YLL, SMS, and HKY carried out the molecular experiments. MJL, YMK, JEJ, and JL researched data and contributed to discussion. J-YP conceived the study and participated in its design and coordination and helped to draft the manuscript. All authors read and approved the final manuscript. J-YP is the guarantor of this manuscript.

\section{Acknowledgement}

This work was supported by a grant (NRF-2014R1A1A1004798) from the Basic Science Research Program (National Research Foundation of Korea funded by the Ministry of Science, Information \& Communication Technology) and Future Planning and by a grant (2011-485 and 2014-122) from the Asan Institute of Life Sciences, Republic of Korea. These funding sources played no role in the study design, collection, analysis, interpretation of data, the writing of the manuscript and the decision to submit the manuscript for publication.

\section{Author details}

${ }^{1}$ Asan Diabetes Center, University of Ulsan College of Medicine, Seoul, Republic of Korea. ${ }^{2}$ Asan Institute of Life Sciences, University of Ulsan College of Medicine, Seoul, Republic of Korea. ${ }^{3}$ Department of Internal Medicine, Asan Medical Center, University of Ulsan College of Medicine,

Poongnap-dong, Songpa-gu, Seoul 138-736, Republic of Korea. 
Received: 20 January 2015 Accepted: 21 March 2015

\section{Published online: 15 April 2015}

\section{References}

1. Hotamisligil GS. Inflammation and metabolic disorders. Nature. 2006;444 (7121):860-7.

2. Yancopoulos GD, Davis S, Gale NW, Rudge JS, Wiegand SJ, Holash J. Vascular-specific growth factors and blood vessel formation. Nature. 2000;407(6801):242-8.

3. Hato $T$, Tabata M, Oike $Y$. The role of angiopoietin-like proteins in angiogenesis and metabolism. Trends Cardiovasc Med. 2008;18(1):6-14.

4. Kadomatsu T, Endo M, Miyata K, Oike Y. Diverse roles of ANGPTL2 in physiology and pathophysiology. Trends Endocrinol Metab. 2014;25(5):245-54.

5. Kadomatsu T, Tabata M, Oike Y. Angiopoietin-like proteins: emerging targets for treatment of obesity and related metabolic diseases. FEBS J. 2011;278(4):559-64.

6. Kim I, Moon SO, Koh KN, Kim H, Uhm CS, Kwak HJ, et al. Molecular cloning, expression, and characterization of angiopoietin-related protein. angiopoietin-related protein induces endothelial cell sprouting. J Biol Chem. 1999;274(37):26523-8.

7. Santulli G. Angiopoietin-like proteins: a comprehensive look. Front Endocrinol. 2014;5:4.

8. Zheng J, Umikawa M, Cui C, Li J, Chen X, Zhang C, et al. Inhibitory receptors bind ANGPTLs and support blood stem cells and leukaemia development. Nature. 2012;485(7400):656-60.

9. Horio E, Kadomatsu T, Miyata K, Arai Y, Hosokawa K, Doi Y, et al. Role of endothelial cell-derived angpt12 in vascular inflammation leading to endothelial dysfunction and atherosclerosis progression. Arterioscler Thromb Vasc Biol. 2014;34(4):790-800,

10. Tabata M, Kadomatsu T, Fukuhara S, Miyata K, Ito Y, Endo M, et al. Angiopoietin-like protein 2 promotes chronic adipose tissue inflammation and obesity-related systemic insulin resistance. Cell Metab. 2009;10(3):178-88.

11. Thorin-Trescases N, Thorin E. Angiopoietin-like-2: a multifaceted protein with physiological and pathophysiological properties. Expert Rev Mol Med. 2014;16:e17.

12. Tian Z, Miyata K, Tazume H, Sakaguchi H, Kadomatsu T, Horio E, et al. Perivascular adipose tissue-secreted angiopoietin-like protein 2 (Angpt12) accelerates neointimal hyperplasia after endovascular injury. J Mol Cell Cardiol. 2013;57:1-12.

13. Tazume H, Miyata K, Tian Z, Endo M, Horiguchi H, Takahashi O, et al. Macrophage-derived angiopoietin-like protein 2 accelerates development of abdominal aortic aneurysm. Arterioscler Thromb Vasc Biol. 2012;32(6):1400-9.

14. Farhat N, Thorin-Trescases N, Mamarbachi M, Villeneuve L, Yu C, Martel C, et al. Angiopoietin-like 2 promotes atherogenesis in mice. J Am Heart Assoc. 2013;2(3):e000201.

15. O'Leary DH, Polak JF, Kronmal RA, Manolio TA, Burke GL, Wolfson Jr SK. Carotid-artery intima and media thickness as a risk factor for myocardial infarction and stroke in older adults. Cardiovascular Health Study Collaborative Research Group. N Engl J Med. 1999;340(1):14-22.

16. Jung $\mathrm{CH}$, Lee MJ, Kang YM, Jang JE, Leem J, Lee YL, et al. Association of serum C1q/TNF-related protein-9 concentration with arterial stiffness in subjects with type 2 diabetes. J Clin Endocrinol Metab. 2014;99(12):E2477-84.

17. Okita K, Iwahashi H, Kozawa J, Okauchi Y, Funahashi T, Imagawa A, et al. Homeostasis model assessment of insulin resistance for evaluating insulin sensitivity in patients with type 2 diabetes on insulin therapy. Endocr $J$. 2013;60(3):283-90.

18. Emoto M, Nishizawa Y, Maekawa K, Hiura Y, Kanda H, Kawagishi T, et al. Homeostasis model assessment as a clinical index of insulin resistance in type 2 diabetic patients treated with sulfonylureas. Diabetes Care. 1999;22(5):818-22.

19. Levey AS, Bosch JP, Lewis JB, Greene T, Rogers N, Roth D. A more accurate method to estimate glomerular filtration rate from serum creatinine: a new prediction equation. Modification of Diet in Renal Disease Study Group. Ann Intern Med. 1999;130(6):461-70.

20. Yugar-Toledo JC, Bonalume Tacito LH, Ferreira-Melo SE, Sousa W, Consolin-Colombo F, rrigoyen MC, et al. Low-renin (volume dependent) mild-hypertensive patients have impaired flow-mediated and glyceryl-trinitrate stimulated vascular reactivity. Circ J. 2005;69(11):1380-5.

21. Lonn E. Use of carotid ultrasound to stratify risk. Can J Cardiol. 2001;17 Suppl A:22A-5

22. Nelson MR, Stepanek J, Cevette M, Covalciuc M, Hurst RT, Tajik AJ. Noninvasive measurement of central vascular pressures with arterial tonometry: clinical revival of the pulse pressure waveform? Mayo Clin Proc. 2010;85(5):460-72.
23. Gonzalez-Juanatey C, Vazquez-Rodriguez TR, Miranda-Filloy JA, Gomez-Acebo I, Testa A, Garcia-Porrua C, et al. Anti-TNF-alpha-adalimumab therapy is associated with persistent improvement of endothelial function without progression of carotid intima-media wall thickness in patients with rheumatoid arthritis refractory to conventional therapy. Mediators Inflamm. 2012;2012:674265.

24. Oh SW. Obesity and metabolic syndrome in Korea. Diabetes Metab J. 2011;35(6):561-6.

25. Usui T, Ninomiya T, Nagata M, Takahashi O, Doi Y, Hata J, et al. Angiopoietin-like protein 2 is associated with chronic kidney disease in a general Japanese population: the Hisayama Study. Circ J. 2013;77(9):2311-7.

26. Meng QX, Wen L, Chen XY, Zhong HJ. Association of serum angiopoietin-like protein 2 and epinephrine levels in metabolically healthy but obese individuals: and evidence. Exp Ther Med. 2013;5(6):1631-6.

27. Li Q, Gong W, Yang Z, Lu B, Yang Y, Zhao W, et al. Serum Angptl2 levels are independently associated with albuminuria in type 2 diabetes. Diabetes Res Clin Pract. 2013;100(3):385-90.

28. Kawashima S, Yokoyama M. Dysfunction of endothelial nitric oxide synthase and atherosclerosis. Arterioscler Thromb Vasc Biol. 2004;24(6):998-1005.

29. Oike $Y$, Tabata M. Angiopoietin-like proteins-potential therapeutic targets for metabolic syndrome and cardiovascular disease. Circ J. 2009:73(12):2192-7.

30. Kitazawa M, Nagano M, Masumoto KH, Shigeyoshi Y, Natsume T, Hashimoto S. Angiopoietin-like 2, a circadian gene, improves type 2 diabetes through potentiation of insulin sensitivity in mice adipocytes. Endocrinology. 2011;152(7):2558-67.

31. Doi Y, Ninomiya T, Hirakawa Y, Takahashi O, Mukai N, Hata J, et al. Angiopoietin-like protein 2 and risk of type 2 diabetes in a general Japanese population: the Hisayama study. Diabetes Care. 2013;36(1):98-100.

32. Ota T. Chemokine systems link obesity to insulin resistance. Diabetes Metab J. 2013;37(3):165-72.

33. Ford ES. Body mass index, diabetes, and C-reactive protein among U.S. adults. Diabetes Care. 1999;22(12):1971-7.

34. Visser M, Bouter LM, McQuillan GM, Wener MH, Harris TB. Elevated C-reactive protein levels in overweight and obese adults. JAMA. 1999;282(22):2131-5.

35. Van Gaal LF, Mertens IL, De Block CE. Mechanisms linking obesity with cardiovascular disease. Nature. 2006;444(7121):875-80.

36. Park HS, Park JY, Yu R. Relationship of obesity and visceral adiposity with serum concentrations of CRP, TNF-alpha and IL-6. Diabetes Res Clin Pract. 2005;69(1):29-35.

37. Anty R, Bekri S, Luciani N, Saint-Paul MC, Dahman M, lannelli A, et al. The inflammatory C-reactive protein is increased in both liver and adipose tissue in severely obese patients independently from metabolic syndrome, Type 2 diabetes, and NASH. Am J Gastroenterol. 2006;101(8):1824-33.

38. Calabro P, Chang DW, Willerson JT, Yeh ET. Release of C-reactive protein in response to inflammatory cytokines by human adipocytes: linking obesity to vascular inflammation. J Am Coll Cardiol. 2005;46(6):1112-3.

39. Muramoto A, Tsushita K, Kato A, Ozaki N, Tabata M, Endo M, et al. Angiopoietinlike protein 2 sensitively responds to weight reduction induced by lifestyle intervention on overweight Japanese men. Nutr Diabetes. 2011;1:e20.

40. Hajer GR, van Haeften TW, Visseren FL. Adipose tissue dysfunction in obesity, diabetes, and vascular diseases. Eur Heart J. 2008;29(24):2959-71.

\section{Submit your next manuscript to BioMed Central and take full advantage of:}

- Convenient online submission

- Thorough peer review

- No space constraints or color figure charges

- Immediate publication on acceptance

- Inclusion in PubMed, CAS, Scopus and Google Scholar

- Research which is freely available for redistribution 